Invited editorial

\title{
Landmark Study Raises the Bar for Interventional Oncology
}

Authors: Jay Shiao, Helen Winter, and Ricky A. Sharma*

Oncology Department, Old Research Campus Research Building, University of Oxford, Oxford OX3 $7 D Q, U K$

\section{* Correspondence:}

Professor Ricky A. Sharma, NIHR Oxford Biomedical Research Centre, Oncology Department, Old Research Campus Research Building, University of Oxford, Oxford OX3 7DQ, UK.

Tel. 441865617330

E-mail: ricky.sharma@oncology.ox.ac.uk 
The recent publication of the SIRFLOX trial in the Journal of Clinical Oncology ${ }^{1}$ is a landmark for oncologists and radiologists involved in the rapidly advancing field of Interventional Oncology and liver-directed therapies. The findings are relevant to all oncologists who treat patients with liver metastases.

Although previous, smaller studies have demonstrated impressive clinical outcomes for patients with metastatic colorectal cancer ( $\mathrm{mCRC}$ ) from combining selective internal radiation therapy (SIRT) with fluoropyrimidine-based chemotherapy ${ }^{2,3}$, SIRFLOX represents the first report of a large-scale randomised trial of this combinatorial therapy. In SIRFLOX, patients received concomitant chemotherapy with 5-fluorouracil and oxaliplatin (FOLFOX), the scientific rationale being that both agents potentially radiosensitise the liver metastases to $\mathrm{SIRT}^{4}$.

SIRFLOX was a randomised, controlled trial of FOLFOX chemotherapy with or without the addition of yttrium-90 resin microspheres (SIR-Spheres) as first-line treatment of patients with liver-only or liver-dominant mCRC. The trial was conducted at 87 sites in Australia, United States of America, Europe, Israel and New Zealand. The study recruited 530 patients from October 2006 to April 2013, 263 patients in the control arm (who received mFOLFOX6 chemotherapy) and the remainder in the test arm (combination of the same chemotherapy, with a dose modification of oxaliplatin for 3 cycles, plus SIRT with cycle 1). Bevacizumab was permitted at the investigator's discretion, but could only be added from cycle 4 of chemotherapy onwards for patients in the test arm.

Patient selection criteria included World Health Organization performance status of 0 or 1 , liver metastases deemed unresectable, life expectancy of $\geq 3$ months, limited extrahepatic metastases in the lung (fewer than five nodules of $\leq 1 \mathrm{~cm}$ diameter or a single nodule of $\leq 1.7 \mathrm{~cm}$ diameter) and/or lymph node involvement in a single anatomic area of $<2 \mathrm{~cm}$ diameter. Of note, $46 \%$ and $44.6 \%$ of patients in the control arm and treatment arm respectively had the primary tumour in situ and 88.6\% and $90.3 \%$ in the control and treatment arm respectively had synchronous presentation of liver metastases and primary tumour (as opposed to metachronous). It is generally recognised that synchronous presentation of liver metastases ${ }^{5,6}$ is a as negative prognostic factor for patients with $\mathrm{mCRC}$, meaning that the subject population of the SIRFLOX study represented the worse prognosis end of the mCRC spectrum, albeit only patients with liver-only or liver-dominant disease.

The first notable achievement of SIRFLOX is the recruitment rate and completion of target recruitment. The investigators must be highly commended in this regard since it is exceptional in the field of Interventional Oncology. Previous interventional studies have failed to recruit to target ${ }^{7}$, and contemporary radiotherapy studies have had to combine datasets from inadequately recruiting studies in order to achieve statistical significance. ${ }^{8}$ It should be mentioned here that SIRT is delivered by a broad multi-disciplinary team, requiring expertise in vascular angiography, radiation dosing, complex imaging, radiation protection, nuclear medicine, medical physics and, in these patients, systemic therapy for metastatic disease. SIRFLOX demonstrates that well-designed interventional oncology studies requiring extensive multi-disciplinary working can achieve respectable recruitment rates and achieve target recruitment despite the potential obstacles.

The primary endpoint of SIRFLOX was progression-free survival (PFS). Median PFS statistics in the control arm and test arm were 10.2 and 10.7 months respectively; the primary endpoint of the study aiming to show the superiority of the novel treatment combination was therefore not achieved. Importantly for SIRT as a liver-directed therapy, the study's secondary endpoint, PFS in the liver, was highly significant with a hazard ratio of $0.69(P=0.002)$. The key message from this endpoint was that the addition of SIR-Spheres $\mathrm{Y}-90$ resin microspheres to standard first-line chemotherapy prolonged PFS in the liver to 20.5 months, compared to 12.6 months in the control group. With regard to other 
secondary endpoints, complete response rate in the liver (1.9\% versus $6.0 \%, \mathrm{P}=0.020)$ and the objective response rate $(68.8 \%$ versus $78.7 \% ; \mathrm{P}=.042)$, i.e. the proportion of patients with complete or partial response, both defined by RECIST version 1.0, were significantly different between the control versus the treatment arm.

The question provoked by this impressive result in the secondary endpoints of the study is whether or not a highly significant effect on liver-specific PFS will equate to an effect on overall survival (OS) for patients with $\mathrm{mCRC}$ ? This is a key question that matters to patients with this disease. Unfortunately, OS results are not yet available from SIRFLOX. We therefore have to consider other forms of liver-directed therapy to speculate what the answer might be when it is eventually reported. Although a head-to-head trial never has been performed and never will be performed, there is general agreement that successful liver surgery on metastases from CRC improves a patient's chances of being alive more than 3 years from the diagnosis of $\mathrm{mCRC}$. In the seminal European EORTC study of liver surgery with or without chemotherapy, median OS for 114 patients treated with surgery (and no chemotherapy) was in excess of 54 months, which is considerably longer than the median statistics for $\mathrm{MCRC}$ patients treated with chemotherapy alone and no surgery in other studies. ${ }^{9,10}$ In the EORTC CLOCC study of the addition of radiofrequency ablation (RFA) added to chemotherapy in 119 patients with up to 9 liver-only metastases from mCRC, a significant effect on PFS did equate to a highly significant OS benefit. ${ }^{7,11}$ These two EORTC studies suggest that successful locoregional treatment of liver metastases in patients with mCRC can confer an OS benefit in carefully selected patient groups. Whether or not the subject group in SIRFLOX turns out to be as carefully selected as these other studies remains to be proven.

Importantly for patients considering SIRT as a treatment option, the SIRFLOX study provides systematic and reliable data on the safety of this complex intervention in patients with $\mathrm{mCRC}$ receiving FOLFOX chemotherapy. Interestingly, the study included some low-volume SIRT centres which were relatively new to the procedure. Previous concerns about using SIRT in combination with radiosensitising chemotherapy (FOLFOX) were allayed by the safety profile reported in the SIRFLOX publication. ${ }^{1}$ Grade 3 and 4 adverse events related to SIRT included gastric ulceration in 9 patients, two of whom required surgical intervention, and radiation-induced liver disease (RILD) in 5 patients. Two patients out of 256 treated with SIRT experienced fatal hepatic failure, one case occurring more than 2 years after SIRT. These figures allow SIRT practitioners worldwide to accurately state the likely incidence of severe side effects from SIRT when it is used in combination with FOLFOX chemotherapy.

In 2017, it is eagerly anticipated that the pre-planned combined analysis of the survival data from the SIRFLOX, FOXFIRE and FOXFIRE-Global studies will be presented. ${ }^{1,12}$ If the positive results of SIRFLOX do indeed herald a survival benefit for the study patients when this is reported next year, the field of interventional oncology will be visibly accelerating the rate of change in the global standard of care for patients with mCRC.

\footnotetext{
${ }^{1}$ Hazel, G. A. Van, V. Heinemann, N. K. Sharma, M. P. N. Findlay, J. Ricke, M. Peeters, D. Perez, B. A. Robinson, A. H. Strickland, T. Ferguson, J. Rodriguez, H. Kroning, I. Wolf, V. Ganju, E. Walpole, E. Boucher, T. Tichler, E. Shacham-Shmueli, A. Powell, P. Eliadis, R. Isaacs, D. Price, F. Moeslein, J. Taieb, G. Bower, V. Gebski, M. Van Buskirk, D. N. Cade, K. Thurston, and P. Gibbs. "SIRFLOX: Randomized Phase III Trial Comparing First-Line MFOLFOX6 (Plus or Minus Bevacizumab) Versus MFOLFOX6 (Plus or Minus Bevacizumab) Plus Selective Internal Radiation Therapy in Patients With Metastatic Colorectal Cancer." Journal of Clinical Oncology (2016): n. pag. Web.
} 


\footnotetext{
2 Van Hazel G, Blackwell A, Anderson J, et al: Randomised phase 2 trial of SIR-Spheres plus fluorouracil/ leucovorin chemotherapy versus fluorouracil/leucovorin chemotherapy alone in advanced colorectal cancer. J Surg Oncol 88:78-85, 2004
}

${ }^{3}$ Sharma RA, Van Hazel GA, Morgan B, et al: Radioembolization of liver metastases from colorectal cancer using yttrium-90 microspheres with concomitant systemic oxaliplatin, fluorouracil, and leucovorin chemotherapy. J Clin Oncol 25:10991106, 200716.

4 Nicolay NH, Berry DP, Sharma RA. Liver metastases from colorectal cancer: radioembolization with systemic therapy. Nat Rev Clin Oncol 2009;6:687-97.

${ }^{5}$ Bova R, Kamphues C, Neuhaus P, Puhl G. [Impact of time of occurrence of liver metastases (synchronous vs. metachronous) on early postoperative outcome and long-term survival of colorectal cancer patients]. Zentralbl Chir. 2014;139:220-225.

${ }^{6}$ Qiao $T, X u Y$, Guan X, Miao D, Wang X. [Analysis of treatment and prognostic factors in colorectal cancer liver metastasis]. Zhonghua Wei Chang Wai Ke Za Zhi. 2015 Sep;18(9):930-4. . PubMed PMID: 26404693.

${ }^{7}$ Ruers, T., C. J. A. Punt, F. Van Coevorden, J.- P. Pierie, I. Borel Rinkes, J. Ledermann, G. Poston, W. Bechstein, M.- A. Lentz, M. Mauer, E. Van Cutsem, M. Lutz, and B. Nordlinger. "O-018* Radiofrequency Ablation (RFA) Combined with Chemotherapy for Unresectable Colorectal Liver Metastases (CRC LM): Long-term Survival Results of a Randomised Phase II Study of the EORTC-NCRI CCSG-ALM Intergroup 40004 (CLOCC)." Annals of Oncology 26.Suppl 4 (2015): Iv114-v115. Web. ${ }^{8}$ Chang JY, Senan S, Paul MA, Mehran RJ, Louie AV, Balter P, Groen HJ, McRae SE, Widder J, Feng L, et al. Stereotactic ablative radiotherapy versus lobectomy for operable stage I non-small-cell lung cancer: a pooled analysis of two randomised trials. Lancet Oncol. 2015 Jun;16(6):630-7. doi: 10.1016/S1470-2045(15)70168-3. Epub 2015 May 13. Erratum in: Lancet Oncol. 2015 Sep;16(9):e427. PubMed PMID: 25981812; PubMed Central PMCID: PMC4489408.

${ }^{9}$ Nordlinger, Bernard, Halfdan Sorbye, Bengt Glimelius, Graeme J. Poston, Peter M. Schlag, Philippe Rougier, Wolf O. Bechstein, John N. Primrose, Euan T. Walpole, Meg Finch-Jones, Daniel Jaeck, Darius Mirza, Rowan W. Parks, Murielle Mauer, Erik Tanis, Eric Van Cutsem, Werner Scheithauer, and Thomas Gruenberger. "Perioperative FOLFOX4 Chemotherapy and Surgery versus Surgery Alone for Resectable Liver Metastases from Colorectal Cancer (EORTC 40983): Long-term Results of a Randomised, Controlled, Phase 3 Trial." The Lancet Oncology 14.12 (2013): 1208-215. Web. ${ }^{10}$ Bokemeyer C, Van Cutsem E, Rougier P, Ciardiello F, Heeger S, Schlichting M, Celik I, Köhne CH. Addition of cetuximab to chemotherapy as first-line treatment for KRAS wild-type metastatic colorectal cancer: pooled analysis of the CRYSTAL and OPUS randomised clinical trials. Eur J Cancer. 2012 Jul;48(10):1466-75. doi: 10.1016/j.ejca.2012.02.057. Epub 2012 Mar 23. PubMed PMID: 22446022.

${ }^{11}$ Ruers T, Punt C, Van Coevorden F, Pierie JP, Borel-Rinkes I, Ledermann JA, Poston G, Bechstein W, Lentz MA, Mauer M, Van Cutsem E, Lutz MP, Nordlinger B; EORTC Gastro-Intestinal Tract Cancer Group, Arbeitsgruppe Lebermetastasen undtumoren in der Chirurgischen Arbeitsgemeinschaft Onkologie (ALM-CAO) and the National Cancer Research Institute Colorectal Clinical Study Group (NCRI CCSG). Radiofrequency ablation combined with systemic treatment versus systemic treatment alone in patients with non-resectable colorectal liver metastases: a randomized EORTC Intergroup phase II study (EORTC 40004). Ann Oncol. 2012 Oct;23(10):2619-26. Epub 2012 Mar 19. PubMed PMID: 22431703; PubMed Central PMCID: PMC3457746.

12 Dutton SJ, Kenealy N, Love SB, Wasan HS, Sharma RA; FOXFIRE Protocol Development Group and the NCRI Colorectal Clinical Study Group. FOXFIRE protocol: an open-label, randomised, phase III trial of 5-fluorouracil, oxaliplatin and folinic acid (OxMdG) with or without interventional Selective Internal Radiation Therapy (SIRT) as first-line treatment for patients with unresectable liver-only or liver-dominant metastatic colorectal cancer. BMC Cancer. 2014 Jul 9;14:497. doi: 10.1186/1471-2407-14-497. PubMed PMID: 25011439; PubMed Central PMCID: PMC4107961. 